\title{
Training on reporting animal welfare concerns
}

\author{
Shannon Reynolds, CPIA, RLAT
}

As mentioned in the inaugural Fruits of Education column, regulatory bodies with oversight of animal care and use have placed an increased emphasis on training along with additional expectations. In the past year, we have seen this emphasis in the adoption of the 8th edition of the Guide for the Care and Use of Laboratory Animals by the Office of Laboratory Animal Welfare and in the issuance of the final rule pertaining to contingency plans by the US Department of Agriculture's Animal and Plant Health Inspection Service.

Training on the reporting of animal welfare concerns might seem a basic and simple principle of research education, but if we don't get this right, the potential impact to our animal care program can be substantial. People will not report concerns if they don't know how, and the IACUC cannot address problems it doesn't know about. But the real training opportunity is building communication such that questions and concerns can be raised and discussed in a context of fairness and transparency. The goal is a culture in which everyone can bring ideas or questions forward, toward the aims of ongoing program improvement and refinement.

It's not uncommon for someone to be uncertain whether a concern should be reported, which could result in a critical delay. I've often heard individuals begin with the caveat, "I'm not sure if this is an animal welfare concern, but...." Helping people to recognize that it's okay to have that conversation is an important first step in opening communication. In my experience, the process is intimidating for those who have limited interaction with the IACUC. In some cases, there may be peer pressure to not bring information forward. For these reasons, training on the mechanisms of reporting can ensure that every employee knows what to do about an animal welfare concern.

As trainers, we must take the regulations and requirements for training and integrate them to serve all stakeholders of an animal care and use program. Animal caretakers, investigators, technicians and IACUC/ethics committee members all have distinct roles and responsibilities. Coordination is critical to the ultimate goal of assuring humane and ethical animal care in the performance of important scientific research. To be sure, the challenge faces trainers daily as they develop curricula and training programs in a variety of research settings. We have a wealth of information to share, but we must consider what the trainees truly need from us.

In regard to reporting, an effective training program must address the following questions.

What is a concern? Provide examples and guidance on reporting; emphasize raising concerns proactively with the goal of preventing more serious problems.

Who can report? Discuss the importance of each individual's willingness to take responsibility for reporting a concern or asking a question. Let employees know that they can report concerns on behalf of others.

How to report? Review each option available for reporting concerns, and specifically note confidentiality and anonymity.

Where to go with a concern? Identify members of the IACUC and other points of contact; list the locations of offices and confidential drop-boxes; and provide contact information relevant to the program's process.

What happens afterward? Explain the process for conducting investigations, including reporting requirements; how conflicts of interest and confidentiality will

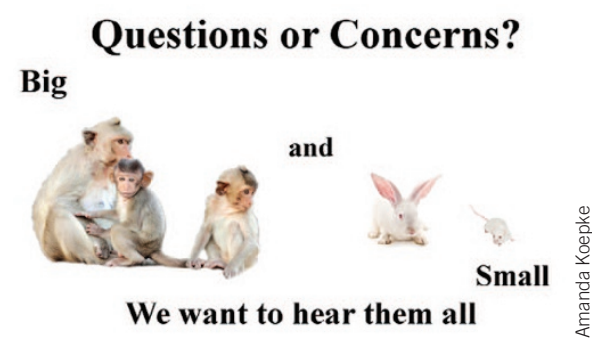

be addressed; and Animal Welfare Act and anti-harassment protections.

Why is reporting important? Discuss how proactive reporting creates opportunities for program refinement and improved animal welfare.

In addition, training the members of the IACUC or ethics committee about investigating reports of animal welfare concerns can be helpful in ensuring that the process is being done fairly and confidentially. A guideline for conducting interviews and reporting outcomes should be part of this training. Many factors must be considered and evaluated with every reported concern, such as whether there is a need for veterinary assessment of an animal's condition, whether any regulatory body or funding agency should be informed and whether a performance issue or violation of employment policy may have occurred. A written process for assessing a concern, notifying the Institutional Official, selecting an impartial investigative team and assessing reporting requirements is useful in communicating the institution's response process to animal care staff, technicians and researchers.

Every training session is an opportunity to build both a stronger animal care culture and a foundation for future refinements. There is considerable value in actively building communication and participation with all team members and stakeholders. 